ARTIGO ORIGINAL

\title{
COMPARAÇÃO DO EFEITO DE UM PROTOCOLO DE CIRCUIT TRAINING EM POLICIAIS MILITARES COM/SEM EQUIPAMENTOS
}

Comparison of the effect of a circuit training protocol on military police with / without equipment

Leonardo A. Nunes ${ }^{1}$, Thiago R. Barbosa ${ }^{1}$, José E. C. Neto ${ }^{1}$, Bráulio N. Lima ${ }^{3,4}$, Leandro B. Camargo ${ }^{3,4}$,

\section{RESUMO}

A Polícia Militar não é apenas uma organização de prevenção e repressão do crime, mas sim uma agência pública de prestação de serviços e de socorro comunitário, onde a segurança da população exige que cada Policial Militar possua um alto grau de aptidão física. O Circuit Training (CT) é considerado como um "sistema de organização dos exercícios para o treinamento de determinadas capacidades e determinados aperfeiçoamentos de habilidade motora como: agilidade, velocidade, força e coordenação". O objetivo deste estudo foi avaliar e comparar a Massa Corporal (MC) os dados gerados a partir do teste de Shuttle Run (SR) antes e após a intervenção do programa de 12 semanas de CT. A amostra deste estudo foi composta por 40 policiais militares do sexo masculino divididos em quatro grupos para a realização do teste SR: controle com (CC) e sem (CS) equipamento; intervenção com (IC) e sem (IS) equipamentos. O protocolo de CT foi composto por 10 estações de exercícios, onde o participante deveria permanecer em cada estação por 30 segundos para dar 3 voltas; após cada volta 5 minutos de intervalo ativo; as seções seguiram com uma frequência semanal de 3 vezes durante 12 semanas. O teste SR foi utilizado para avaliar performance na semana anterior e posterior ao protocolo de intervenção de CT. A MC foi aferida antes da aplicação do teste SR. Os resultados sugerem que o protocolo de 12 semanas de CT adotado para esse estudo gerou diferenças consideradas estatisticamente significantes ( $\mathrm{P} \leq 0,05)$, diminuindo a $\mathrm{MC}$ e o tempo no teste $\mathrm{SR}$ dos grupos intervenção (IC e IS) nas condições pós intervenção em relação as condições pré intervenção e controle. A utilização de equipamentos pelo grupo IC não interferiu no resultado e não gerou diferenças consideradas estatisticamente significantes em relação ao grupo IS. O presente trabalho apresenta o CT como uma ferramenta aplicável e geradora de resultados satisfatórios para policiais militares.

Palavras-chave: Agilidade; Circuit Training; Shuttle Run; Policiais Militares; Avaliação.

\section{ABSTRACT}

The Military Police is not just a crime prevention and repression organization, but a public service and community relief agency, where the safety of the population requires every Military Policeman to have a high degree of physical fitness. Circuit Training (CT) is considered to be a "training organization system for training certain skills and certain motor skill enhancements such as agility, speed, strength and coordination". The aim of this study was to evaluate and compare Body Mass (MC) data generated from the Shuttle Run (SR) test before and after the intervention of the 12week CT program. The study sample consisted of 40 male military police officers divided into four groups to perform the SR test: control with (CC) and without (CS) equipment; intervention with (CI) and without (IS) equipment. The CT protocol consisted of 10 exercise stations, where the participant should remain in each station for 30 seconds to do 3 laps; after each lap 5 minutes of active break; the sections followed a weekly frequency of 3 times for 12 weeks. The SR test was used to evaluate performance in the week before and after the TC intervention protocol. The MC was measured before applying the SR test. The results suggest that the 12-week CT protocol adopted for this study generated differences considered statistically significant $(\mathrm{P} \leq 0.05)$, decreasing the $\mathrm{MC}$ and time in the SR test of the intervention groups (CI and IS) in the post-intervention conditions. relation to pre intervention and control conditions. The use of equipment by the CI group did not interfere in the result and did not generate differences considered statistically significant in relation to the IS group. This paper presents the TC as an applicable tool and satisfactory result generator for military police.

Keywords: Circuit Training; Shuttle Run; Military police officers; Body mass; Evaluation.

Autor de correspondência

Klebson Almeida - Universidade da Amazônia UNAMA), Campus Alcindo Cacela - Av. Alcindo Cacela, 287 - Umarizal Belém-PA Bloco 'D', $5^{\circ}$ andar - CEP: 66.06-902 Belém (PA), Brasil.
${ }^{1}$ Universidade da Amazônia, Centro de Ciências Biológicas e da Saúde, Belém, PA.

${ }^{2}$ NUTRESPORTE, Exercícios, Reabilitação e Nutrição para o Esporte.

${ }^{3}$ Universidade Metodista de Piracicaba, UNIMEP.

${ }^{4}$ Núcleo de Pesquisas em Bomecânica Ocupacional e Qualidade de Vida, NPBOQV. 


\section{INTRODUÇÃO}

Durante a vinda, em 1808, da Família Real Portuguesa foram criadas no Brasil as instituições militares e apenas em 1946 receberam a denominação de Polícias Militares (PMs), que tinham como missão a segurança interna e a manutenção da ordem. A Policia Militar (PM) brasileira mais antiga é a de Minas Gerais - Dragão de Minas em 1775, em seguida a do Rio de Janeiro, depois a do Pará em $1818^{(1)}$.

A Polícia Militar não é apenas uma organização de prevenção e repressão do crime, mas sim uma agência pública de prestação de serviços e de socorro comunitário, onde a segurança da população exige que cada Policial Militar possua um alto grau de aptidão física. O Policial Militar necessita de um mínimo de aptidão física para desenvolver as tarefas do dia-a-dia. Contudo, este não precisa ser um atleta. $\mathrm{O}$ Teste de aptidão física (TAF) utilizado na corporação não tem finalidade competitiva, mas sim de ser um instrumento de diagnóstico para fins de seleção e de verificação dos níveis de aptidão dos integrantes da $\mathrm{PM}^{(2)}$.

A Aptidão Física Relacionada à Saúde “(AFRS) pode ser entendida como sendo a capacidade de realizar as tarefas diárias com vigor sem demonstrar traços e características de esgotamento"(3). Este conceito derivou basicamente de estudos clínicos que evidenciaram maior incidência de problemas de saúde entre adultos e idosos de vida sedentária ${ }^{(4)}$. O Departamento de Saúde e Serviços Humanos dos Estados Unidos - USDHHS diz que "a AFRS inclui a aptidão cardiorrespiratória, força e resistência muscular, composição corporal e flexibilidade"(5).

O Circuit Training (CT) é considerado um sistema de organização dos exercícios para o treinamento de determinadas capacidades e de habilidades motoras, tais como agilidade, velocidade, força e coordenação, constituindo uma forma confiável e eficaz de trabalho. O modelo de CT possui registros de sua utilização na antiguidade, mesmo que aplicado de uma forma diferente da atualidade. Os gregos e romanos deixaram registros que comprovam terem utilizado essa forma de treinamento baseada em $\mathrm{CT}^{(6)}$.

A agilidade é uma das variáveis do desempenho motor mais importante para a Educação Física, sendo capaz de influenciar diretamente capacidades físicas como a força, velocidade, flexibilidade e coordenação. O desenvolvendo da agilidade no individuo, aumenta o domínio do corpo, consequentemente a confiança para a execução do movimento. Testes envolvendo a agilidade são muito utilizadas para compor o TAF, principalmente nas avaliações de atletas de várias modalidades e de Militares que necessitam de atividade cotidiana para exercer seu trabalho $^{(7)}$.

$\mathrm{O}$ treinamento CT se caracteriza pela ordem sucessiva de várias estações de exercícios praticadas em um ou mais circuitos ou voltas, cuja combinação vai possibilitar a obtenção de determinado objeti$\mathrm{vo}^{(8)}$.

É fundamental a compreensão dos resultados obtidos através dos protocolos de treinamento, em especial quando se trata de melhorar a performance de profissionais que necessitam de um bom condicionamento para o desenvolvimento de suas atividades. O presente trabalho pode contribuir com uma proposta de modelo de treinamento para policiais mitares.

O objetivo deste estudo foi avaliar e comparar a Massa Corporal (MC) os dados gerados a partir do teste de Shuttle Run (SR) antes e após a intervenção do programa de 12 semanas de CT, em 04 grupos de policiais militares do $24^{\circ}$ BPMPA com e sem equipamento completo (uniforme completo, colete balístico, coldre e armamento de porte) e grupo controle.

\section{MÉTODOS}

Foi um estudo experimental do tipo longitudinal, prospectivo realizado com policiais militares do 24 Batalhão de Polícia Militar do Estado do Pará (24을 ${ }^{\circ}$ PA) (total de 40 policiais) na quadra do polo esportivo da Paróquia Igreja Nossa Senhora do Bom Remédio, localizada no Conjunto Satélite WE $06 \mathrm{~N}^{\circ}$ 455, Coqueiro - Belém/PA, CEP 66670-420. O projeto desse trabalho foi submetido e aprovado pelo Comitê de Ética e Pesquisa com Seres Humanos da Universidade da Amazônia (UNAMA) sob o número do parecer 2.825.136 (CAAE: 94989818.0.0000.5173).

Os 40 participantes da pesquisa receberam informações sobre os procedimentos os quais seriam submetidos e voluntariamente assinaram o Termo de Consentimento Livre e Esclarecido em conformidade com as instruções contidas na Resolução 466/12 do Concelho Nacional de Saúde para estudos com seres humanos, do Ministério da Saúde.

\section{Participantes}

A amostra deste estudo foi composta por 40 policiais militares, do sexo masculino e adultos. As características idade, estatura e massa corporal (MC) dos participantes deste estudo estão descritas na Tabela 1 .

Os participantes foram divididos em quatro grupos: Grupo controle com equipamentos (CC = 10) e grupo controle sem equipamentos $(C S=10)$, 
Tabela 1 Características da amostra

\begin{tabular}{lcccccccccc}
\hline & \multicolumn{3}{c}{ CS } & \multicolumn{2}{c}{ CC } & & IS & & IC & \\
\hline & Média & DP & Média & DP & Média & DP & Média & DP \\
\cline { 2 - 10 } & 37,80 & $\pm 4,92$ & 36,80 & $\pm 5,57$ & 39,30 & $\pm 5,98$ & 39,60 & $\pm 5,19$ \\
Idade (anos) & 1,72 & $\pm 0,08$ & 1,74 & $\pm 0,06$ & 1,69 & $\pm 0,05$ & 1,69 & $\pm 0,08$ \\
Estatura (m) & 84,08 & $\pm 11,52$ & 82,31 & $\pm 7,58$ & 79,82 & $\pm 5,36$ & 85,16 & $\pm 12,11$ \\
MC pré intervenção (Kg) & & & & & & & & &
\end{tabular}

ambos constituídos por policiais que não efetuarão o protocolo de CT, mantendo a rotina de atividades e participando dos testes SR em condições pré e pós o período de 12 semanas; Grupo intervenção com equipamentos ( $\mathrm{IC}=10)$ e grupo intervenção sem equipamentos (IS $=10$ ), ambos compostos por policiais militares que foram submetidos ao protocolo de CT somado a rotina de atividades e realizando o teste SR nas condições pré e pós o protocolo de intervenção de 12 semanas. Os participantes foram divididos em quatro grupos: Grupo controle com equipamentos $(\mathrm{CC}=10)$ e grupo controle sem equipamentos $(C S=10)$, ambos constituídos por policiais que não efetuarão o protocolo de $\mathrm{CT}$, mantendo a rotina de atividades e participando dos testes SR em condições pré e pós o período de 12 semanas; Grupo intervenção com equipamentos (IC = 10) e grupo intervenção sem equipamentos (IS = 10), ambos compostos por policiais militares que foram submetidos ao protocolo de CT somado a rotina de atividades e realizando o teste SR nas condições pré e pós o protocolo de intervenção de 12 semanas.

\section{Design Experimental}

As avaliações ocorreram em dois encontros, onde o primeiro ocorreu na semana anterior ao início e o segundo na semana posterior ao termino do protocolo de intervenção de CT. No primeiro encontro todos os participantes assinaram o TCLE; responderam a perguntas referentes a condição de saúde e prontidão para a atividade física; tiveram dados, como idade, estatura, massa corporal coletados; foram submetidos ao primeiro teste SR. O protocolo de intervenção consistia em 3 sessões de CT por semana durante 12 semanas. Na semana seguinte ao termino do protocolo de intervenção de CT foi avaliada a massa corporal e foi feito um novo teste SR. Durante o período de aplicação do protocolo de intervenção os grupos intervenção (IC e IS), além do protocolo de CT do estudo, mantiveram suas atividades normais, incluindo uma aula de educação física por semana, enquanto os grupos controles (CC e CS) permaneceram em suas rotinas de atividade ocupacional com apenas uma aula de educação física por semana. $\mathrm{O}$ design experimental está representado no fluxograma da Figura 1.

Os grupos que passaram pelo protocolo de intervenção (IC e IS) executaram o protocolo de CT com roupas de adequadas a prática de atividade física. Os grupos com equipamentos (CC e IC) realizaram o teste SR nas condições pré e pós intervenção utilizando seus equipamentos de trabalho (uniforme completo, colete balístico, coldre e armamento de porte), enquanto os grupos sem equipamentos (CS e IS) realizaram os testes SR com roupas adequadas a prática de atividade física.

Figura 1 Fluxograma do design experimenta.

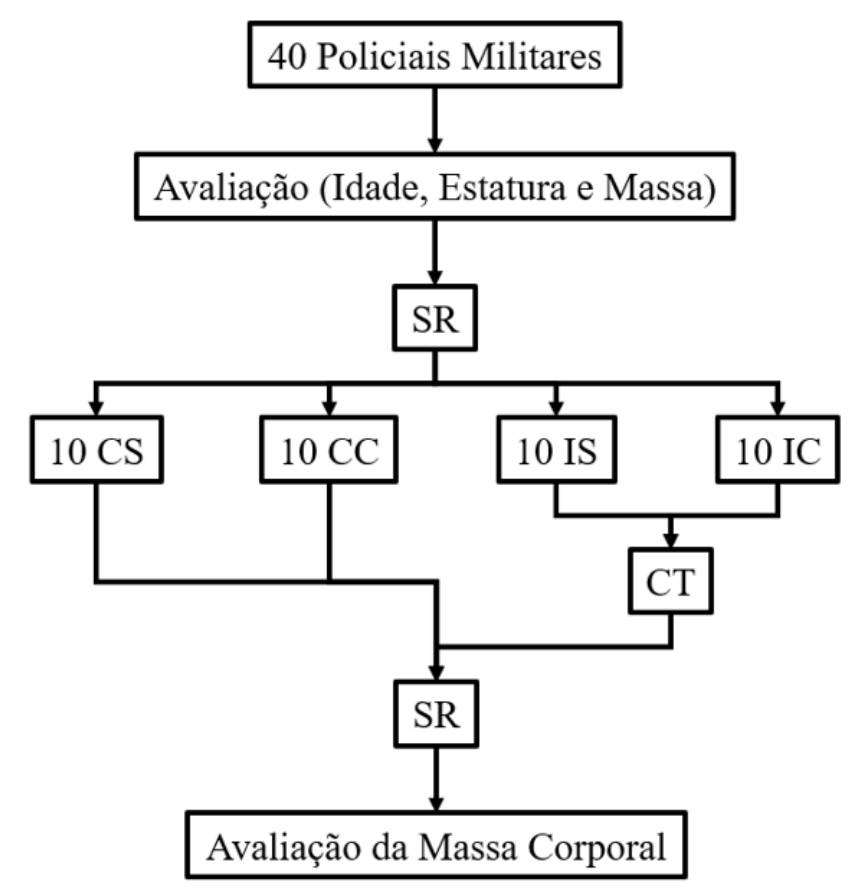

Fonte: Próprio autor.

\section{Protocolo de Intervenção CT}

O protocolo de exercícios CT foi constituído de 10 estações de exercício. Os participantes executaram 30 segundos de cada exercício (estação) com 10 segundos de intervalo para troca. Os participantes fizeram 03 voltas em todas as estações, onde obrigatoriamente precisavam passar em todas as estações para completar 01 volta. Cada volta completada era seguida de 05 minutos de descanso ativo (caminhada 
ou corrida), até completar 03 voltas.

Os exercícios utilizados seguem as nomenclaturas e descrição conforme Aranda e Cases ${ }^{(6)}$. As estações tiveram a seguinte ordem: 1) corrida de vai e volta em obstáculos verticais paralelos, 2) pliometria com mini barreiras + sprint de $3 \mathrm{~m}, 3$ ) lateralidade entre chapéu chinês + escada de agilidade dois pés por vez, 4) polichinelo com corda naval, 5) saltar com um apoio dentro dos arcos, 6) abdominal supra, 7) flexão de braço no solo com apoio fixo, 8) skipping lateral entre estafetas, 9) agachamento e 10) return to the middle "volta para meio"(6,9).

Antes da intervenção do CT os participantes da pesquisa tiveram 05 minutos de aquecimento envolvendo todos os grupos musculares e articulações e, logo após 05 minutos de exercícios de alongamento passivo estático. Após a intervenção do CT, 05 minutos de caminhada de intensidade moderada e leve para retorno da frequência cardíaca e respiratória.

Outras variáveis não foram controladas de forma intencional e por conveniência, entretanto, o grupo de policiais foi escolhido pela facilidade de controle do protocolo e pela homogeneidade em seus cotidianos de treinamento e de atividade física diária. A aplicação do protocolo teve sua ordem randomizada entre os participantes, independente do grupo.

\section{Protocolo de Avaliação SR}

O teste de SR seguiu as recomendações de Hunsicker e Reiff( ${ }^{(10)}$, onde, o participante avaliado, colocou-se o mais próximo possível, porém com os pés atrás da linha de partida. Após o sinal de saída, iniciou-se o teste com o acionamento simultâneo do cronômetro. O participante se desloca em corrida, com à máxima velocidade, até 02 (dois) blocos medindo $5 \times 5 \times 10 \mathrm{~cm}$ cada, dispostos equidistantes, a
9,14 metros da linha de saída. Ao chegar, o participante da pesquisa pegou um dos blocos e retornou ao ponto de partida, depositando esse bloco atrás da linha demarcatória (a mesma também foi utilizada para a partida). O bloco não podia ser jogado, mas sim colocado no solo. Em seguida, sem interromper a corrida, o participante, seguiu em busca do segundo bloco, procedendo da mesma forma. Ao pegar ou deixar o bloco, o participante, transpôs pelo menos com um dos pés as linhas que limitam o espaço de teste. O cronômetro foi parado quando o avaliado colocou o segundo bloco no solo e transpõe com pelo menos um dos pés a linha final ${ }^{(10)}$.

\section{Análise Estatística}

Os dados coletados foram catalogados em fichas protocolares, anexados para alimentar uma planilha no programa Microsoft Office Excel $2010^{\circledR}$ para verificação das médias e desvio padrão e analisados por meio do programa estatístico Statistical Package for the Social Sciences - SPSS, versão 20 para Windows, utilizando o teste Shapiro-Wilk, para verificação da distribuição dos dados e o teste t para comparar grupos e condições. O nível de significância estatística para este estudo será de $5 \%(\mathrm{P} \leq 0,05)$.

\section{RESULTADOS}

Foram avaliados 40 policiais militares do sexo masculino, onde as descrições das características iniciais dos participantes, na condição pré intervenção, já foram apresentadas na Tabela 1. Os valores da massa corporal sofreram alterações após o protocolo de intervenção do CT. Os valores da massa corporal do teste SR nas condições pré e pós intervenção estão apresentados na Tabela 2.

Não foram encontradas diferenças considera-

Tabela 2 Resultados do teste SR em segundos e MC em Kg separados por grupo nas condições pré e pós intervenção.

\begin{tabular}{lcccccccc}
\hline & \multicolumn{2}{c}{ CS } & \multicolumn{2}{c}{ CC } & \multicolumn{2}{c}{ IS } & \multicolumn{2}{c}{ IC } \\
\hline \multirow{2}{*}{ MC pré } & Média & DP & Média & DP & Média & DP & Média & DP \\
\cline { 2 - 9 } MC pós & 84,08 & $\pm 11,52$ & 82,31 & $\pm 7,58$ & $79,82^{*}$ & $\pm 5,36$ & $85,16^{* *}$ & $\pm 12,11$ \\
SR pré & 11,93 & $\pm 0,66$ & 12,06 & $\pm 1,69$ & $12,39^{+}$ & $\pm 0,90$ & $11,98^{++}$ & $\pm 0,59$ \\
SR pós & 12,22 & $\pm 1,71$ & 11,90 & $\pm 0,74$ & $10,97^{+*}$ & $\pm 0,73$ & $10,95^{++*}$ & $\pm 0,72$ \\
\hline
\end{tabular}

Diferenças estatisticamente significantes $(\mathrm{P} \leq 0,05)$ entre: *IS/MC pré e IS/MC pós; **IC/MC pré e IC/MC pós; ${ }^{+}$IS/SR pré e IS/SR pós; ${ }^{++}$IC/SR pré e IC/SR pós; "Valores (IS/SR pós e IC/SRpós) com diferenças estatisticamente significante $(\mathrm{P} \leq 0,05)$ em relação a todas as condições Controle e sem diferenças entre si. Fonte: Próprio autor. 
das estatisticamente significantes entre IS e IC, demonstrando que a utilização do equipamento não interfere na performance do teste SR independente da condição controle, pré ou pós intervenção. As condições pós intervenção (IC/SR pós e IS/SR pós) no teste SR demonstraram diferenças estatisticamente significantes em relação as condições pré intervenção (IC/ SR pré e IS/SR pré). As condições pós intervenção (IC/SR pós e IS/SR pós) no teste SR demonstraram diferenças estatisticamente significantes em relação a todas as condições controle (CC/SR pré, CS/SR pré, CC/SR pós e CS/SR pós). A massa corporal apresentou diferenças estatisticamente significantes entre os grupos pós intervenção (IC/MC pós e IS/MC pós) e todas as demas condições pré (IC/MC pré e IS/MC pré) e controle (CC/MC pré, CS/MC pré, CC/MC pós e CS/MC pós). Os referidos dados gerais estão representados nas Figuras 2.

Figura 2 Gráfico com média e desvio padrão das variáveis SR (s) e MC (Kg) nas condições pré e pós intervenção.

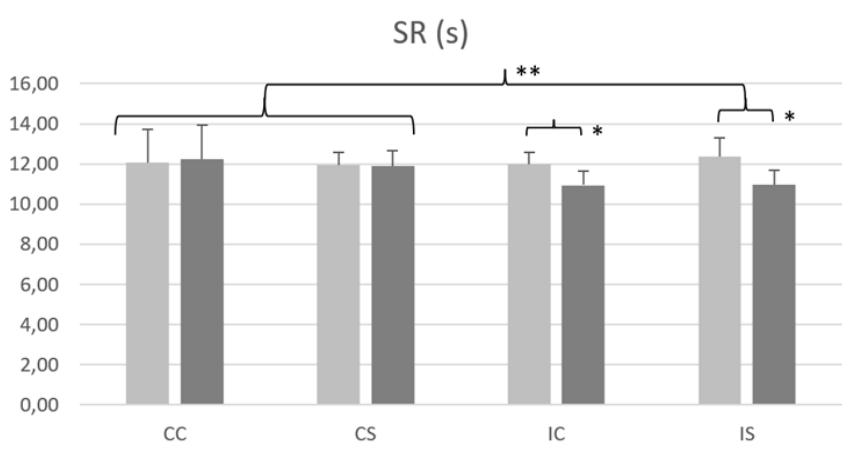

$\mathrm{MC}(\mathrm{Kg})$

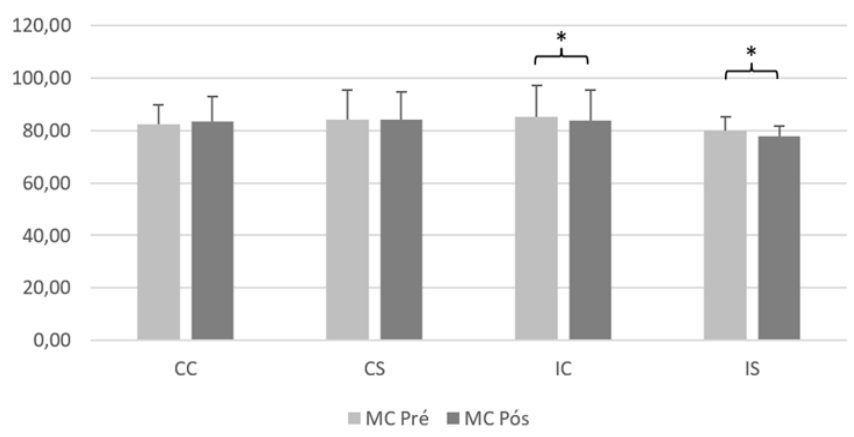

*Diferenças estatisticamente significantes entre as condições pré e pós intervenção; ** Diferenças estatisticamente significantes entre as condições pós intervenção e controle. Fonte: Próprio Autor.

Em uma comparação geral dos 20 participantes do grupo intervenção entre as condições pré e pós intervenção, podemos observar que existe uma diferença estatisticamente significante apresentada na Figura 3.

Tanto os valores de MC quanto de SR tiveram diferenças consideradas estatisticamente significantes entre as condições pré e pós intervenção. O mesmo evento não ocorreu nos grupos controles.

Figura 3 Gráfico com média e desvio padrão das variáveis SR (s) e MC (Kg) contendo todos os 20 participantes dos grupos intervenção (IC e IS) comparando as condições pré e pós intervenção.

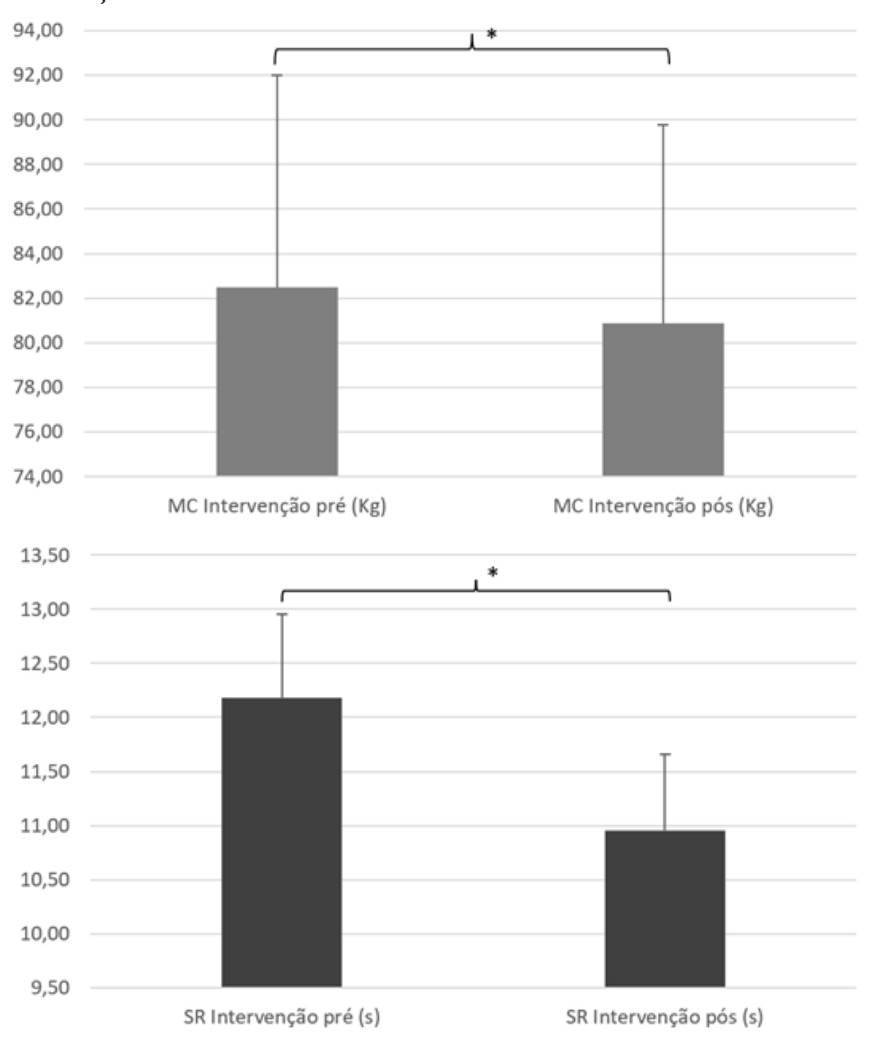

*Diferenças estatisticamente significantes.

Fonte: Próprio autor.

\section{DISCUSSÃO}

Este trabalho teve como objetivo analisar o efeito de um protocolo de 12 semanas de CT na MC e na performance dos policiais militares do $24^{\circ} \mathrm{BPM} /$ PA avaliada através do teste SR. A escolha das variáveis avaliadas, MC e SR, é decorrente do fato de serem fáceis e pouco onerosas para a aplicação em uma rotina de treinamento. Os resultados mostraram-se estatisticamente satisfatórios, apresentando diferenças significantes de diminuição da MC e menor tempo para executar o teste SR.

Atualmente, temos um déficit de policiais militares nos batalhões da região metropolitana de Belém/Pa. De acordo com o último censo realizado pelo IBGE em 2018, a população do Estado do Pará é de 7.581.051, a população de Belém/PA é de 1.393.399 habitantes e a recomendação da ONU é de 01 Policial para cada 250 habitantes ${ }^{(11,12)}$. A Policia Militar do Pará tem 16.243 PM'S em atividade, mas o número de PM'S nas ruas é bem menor de PM'S a disposição nos Batalhões, pois consta neste montante PM'S de outras especialidade, como médico, enfermeiro, psicólogo e os PM'S a disposição de outros órgão que 
necessitam do policiamento ostensivo como é o caso do Judiciário em geral. Parte dos policiais também se encontram afastados pelo departamento médico, com problemas de saúde física ou psicológica. Além do déficit de policiais, a preparação física para que possam exercer suas atividades é pouco estudada demonstrando a importância de estudos como este.

O resultado apresentado na Tabela 2 apresenta analise em cada grupo e em cada condição. Os grupos que foram submetidos a intervenção de 12 semanas de CT obtiveram melhoras, representadas pela diminuição da MC e do tempo de execução do teste SR, demonstrando que o protocolo proposto pode ser considerado eficiente para a melhoria das variáveis estudadas e uma proposta possível de ser inclusa na rotina de treinamento de policiais militares. A utilização dos equipamentos não gerou alterações possíveis de influenciar a significância estatística do estudo entre grupos IC e IS, demostrando que o protocolo de CT pode melhorar a performance mesmo com os equipamentos devido o principio biológico da transferência ${ }^{(13)}$. Franchini e Vecchio ${ }^{(14)}$, enfatizam que a adequada manipulação das mesmas adaptações orgânicas positivas, promove a melhoria das capacidades físicas estimuladas e isso resulta na melhoria do desempenho.

Os grupos controle (CC e CS) não obtiveram diferenças dentro da significância estatística adotada no estudo nas condições pré e pós intervenção, estando este resultado dentro do esperado, pois seguiram em suas rotinas normais de atividades laborais. Por esse motivo, os grupos que sofreram intervenções (IC e IS) na condição pós intervenção obtiveram diferenças estatísticas consideradas significantes em relação aos grupos controle (CC e CS) em todas as condições pré e pós intervenção. Para alguns autores o treinamento em circuito aperfeiçoa todas as aptidões do sistema musculoesquelético um fator que contribui para melhora de cada capacidade física ${ }^{(15)}$.

A Figura 1 demonstra, em gráficos, as diferenças estatisticamente significantes encontradas no estudo, onde foi observado que os tempos do teste SR diminuem após o protocolo de 12 semanas de intervenção de CT, mostrando melhor performance no referido teste. No protocolo, de acordo com a Figura 2, ao comparar todos os participantes dos grupos que foram submetidos (IC e IS) nas condições pré e pós intervenção podemos observar que além da diminuição do tempo no teste SR ainda foi possível observar a diminuição da $\mathrm{MC}$ de acordo com a significância estatística adotada. Como dito anterior mente, outras variáveis, envolvendo rotina e estilo de vida, não foram controladas de forma proposital pois é pretendido identificar se o CT é adequado para a inclusão possibilidades de métodos de treinamento para policiais militares. A rotina dos participantes gera uma característica de homogeneidade no que tange a atuação profissional, portanto neste trabalho é possível de identificar que o protocolo de CT foi eficiente na geração de evidencias de um método de treinamento e de avaliação aplicável para geração de resultados satisfatórios, mesmo com a utilização dos equipamentos (uniforme completo, colete balístico e pistola). É possível que o protocolo de CT tenha influenciado na MC ajudando através do aumento de gasto calórico criando um déficit necessário para a diminuição ${ }^{(16-18)}$.

O presente trabalho corrobora com o fato do treinamento em circuito promover melhorias em todas as capacidades físicas treináveis em poucas semanas $^{(19,20)}$. As melhoras das capacidades físicas se dá através da adaptação neural, especialmente com aumento da sincronização das unidades motora ${ }^{(21)}$. Foi observado que os policiais militares que foram submetidos ao CT obtiveram melhoras. Tendo em vista que todos os militares tiveram apenas um dia de aula de educação física por semana, podemos identificar que o protocolo de CT pode ser incluso na rotina de atividades para melhorar a performance avaliada através de SR e, possivelmente, contribuir com o controle do peso. Podemos afirmar, também, que é fundamental, para os agentes de segurança pública, que estejam ativos fisicamente como forma de preparação para melhor desempenho da função policial militar ${ }^{(2)}$.

\section{CONCLUSÃO}

Os resultados sugerem que o protocolo proposto de 12 semanas de CT pode melhorar a performance de policiais militares avaliados pelo teste SR e ajudar no controle de peso que foi avaliado através da avaliação da MC, pois, a comparação entre as condições pré e pós intervenção demostrou diferenças estatisticamente significantes com a diminuição da MC e do tempo de execução do teste SR. A utilização de equipamentos (uniforme completo, colete balístico e pistola) não foi determinante para diminuir a performance obtidas através do protocolo de CT e avaliado pelo teste SR, o que demonstra que o protocolo adotado pode ser uma ferramenta na rotina de treinamento desses policiais militares. Desta forma podemos considerar o CT como uma excelente ferramenta para aprimoramento da performance avaliada pelo teste SR, que também se trata de um teste de fácil aplicação para a rotina desses profissionais. Contudo a falta de estudos direcionados para a espe- 
cificidade de capacidades físicas e habilidades motoras necessárias para o trabalho rotineiro de policiais militares. Este estudo contribui para a melhoria da forma como policiais militares podem se preparar fisicamente para o exercício de suas funções, apresentando o CT como uma proposta aplicável e geradora de resultados satisfatórios.

\section{AGRADECIMENTOS}

Os acadêmicos agradecem a Deus nosso Senhor pela oportunidade concedida, a Nossa Senhora de Nazaré que intercede por nós junto ao seu Filho Jesus de Nazaré, que nos abençoa todos os dias; ao Professor Mestre em Gerontologia Klebson da Silva Almeida por compartilha o conhecimento e amizade; aos nossos familiares e amigos que também são parte da nossa conquista, sempre nos apoiando nos mostrando o melhor caminho; ao Núcleo de Pesquisar em Biomecânica Ocupacional e Qualidade de Vida pela parceria no desenvolvimento desse manuscrito.

\section{REFERÊNCIAS}

1. Neves GS. A Presença da policial feminina com características afro-descendentes na Polícia Militar da Bahia. Salvador; 2008.

2. Oliveira OI, CAP QOPM, et al. Manual de Educação Física do Piauí. Anexo do Boletim do Comando Geral No 029/2015, Datado de 12/02/20152015.

3. Pate RR. The evolving definition of physical fitness. Quest. 1988;40(3):174-9.

4. Glaner MF. Nível de atividade física e aptidão física relacionada à saúde em rapazes rurais e urbanos. Rev Paul Educ Fís. 2002;16(1):76-85.

5. Department of Health and Human Services US. Physical activity and health: A report of the surgeon general. US Department of Health and Human Services, Centers for Disease Control and ...; 1996.

6. Aranda JS, Cases RS. 1088 Exercícios em circuito2002. 7. Corbin CB, Lindsey R, Welk G. Concepts of physical fitness: Active lifestyles for wellness: McGraw-Hill Boston; 2000 .

8. Guiselini M. Exercícios aeróbicos: teoria e prática no treinamento personalizado e em grupos: Phorte; 2007.

9. Dos Santos MT. Os Efeitos do Treinamento Intervalado e da Pista em Circuito no Desempenho das Atividades de Educação Física do Aluno Policial-Militar. O Alferes. 2016;26(69).

10. Hunsicker PA, Reiff GG. AAPHER. Youth Fitness Test Manual. 1976.

11. Costa HHdC, Farias IS. Aplicação dos Direitos Humanos pelo Policial Militar no Atendimento a Ocorrência. 2019.

12. IBGE IBdGeE. Projeções da População 2018. IBGE Brasília; 2018.

13. Marchetti P, Lopes C, Corrêa D. Planejamento e prescrição do treinamento personalizado: do iniciante ao avançado. São Paulo: Mundo. 2014.

14. Franchini E, Vecchio FBD. Preparação física para atletas de judô2008.
15. Novaes J, Gil A, Rodrigues G. Condicionamento físico e treino funcional: Revisando alguns conceitos e posicionamentos. Revista UNIANDRADE. 2014;15(2):87-93.

16. Girold S, Calmels P, Maurin D, Milhau N, Chatard J-C. Assisted and resisted sprint training in swimming. The Journal of Strength \& Conditioning Research. 2006;20(3):547-54.

17. Moore EWG, Hickey MS, Raoul FR. Comparison of Two Twelve Week Off-Season Combined Training Programs On Entry Level Collegiate Soccer Players'Performance. The Journal of Strength \& Conditioning Research. 2005;19(4):7918.

18. Tricoli V, Lamas L, Carnevale R, Ugrinowitsch C. Short-term effects on lower-body functional power development: weightlifting vs. vertical jump training programs. The Journal of Strength \& Conditioning Research. 2005;19(2):4337.

19. Blazevich AJ, Gill ND, Deans N, Zhou S. Lack of human muscle architectural adaptation after short-term strength training. Muscle \& nerve. 2007;35(1):78-86.

20. Pincivero DM, Lephart SM, Karunakara RG. Effects of rest interval on isokinetic strength and functional performance after short-term high intensity training. British journal of sports medicine. 1997;31(3):229-34.

21. Seynnes OR, de Boer M, Narici MV. Early skeletal muscle hypertrophy and architectural changes in response to high-intensity resistance training. Journal of applied physiology. 2007;102(1):368-73.

\section{OBSERVAÇÕES}

Os autores declaram não existir conflitos de interesse de qualquer natureza.

Os autores Leandro Borelli de Camargo e Ricardo Pablo Passos, são bolsistas da CAPES. 